\title{
AMOUNTS OF GONADOTROPHINS REQUIRED FOR NORMAL FOLLICULAR GROWTH IN HYPOPHYSECTOMIZED ADULT RATS
}

\author{
R. WELSTCHEN \\ Department of Anatomy, \\ Rotterdam Medical Faculty, The Netherlands

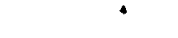

Rats were hypophysectomized at oestrus, at days I, 2 and 3 of dioestrus or at prooestrus. They were injected with various doses of PMS only, or of combinations of PMS and HCG, in such a way that rather constant blood levels could be expected. They were killed 24 hours later.

It was found that the minimal doses of the substitutional gonadotrophins required to maintain normal follicular growth, were considerably higher during the period from prooestrus to oestrus (when a new generation of follicles starts its development) than during all other periods. This peak requirement primarily concerned PMS. The minimal requirement during other periods only showed minor variations.

The data suggest that during prooestrus very high amounts of FSH are required to recruit a new crop of follicles, whereas during the other phases low levels of both FSH and/or LH are a requirement for normal follicular growth.

\author{
QUANTITÉS DE GONADOTROPINES NÉCESSAIRES \\ POUR ASSURER LA CROISSANCE FOLLICULAIRE NORMALE \\ CHEZ DES RATTES ADULTES HYPOPHYSECTOMISÉES
}

L'hypophysectomie est pratiquée à l'cestrus ou les jours I, 2 ou 3 du diœstrus ou au procestrus. Les rattes reçoivent alors des doses variables de PMS seule ou d'une combinaison PMS + HCG. Le traitement est donné de telle façon qu'un niveau sanguin à peu près constant soit maintenu. Les animaux sont abattus $24 \mathrm{~h}$ plus tard.

Les doses minimales de gonadotropines de remplacement nécessaires au maintien d'une croissance folliculaire normale sont considérablement plus élevées pendant la 
période allant du proœstrus à l'œstrus (quand une nouvelle génération de follicules commence à se développer) qu'à tout autre moment. Le besoin gonadotrope important concerne essentiellement PMS. La dose minimale nécessaire pendant les autres stades du cycle ne subit que des modifications peu importantes.

Ces résultats permettent de penser qu'au cours du proœstrus de très grandes quantités de FSH sont nécessaires au recrutement d'un nouveau groupe de follicules, alors que pendant les autres stades du cycle la quantité de FSH et/ou de $L H$ nécessaire pour une croissance normale des follicules est peu élevée. 